Journal of Knot Theory and Its Ramifications

Vol. 25, No. 14 (2016) 1699001 (8 pages)

(C) World Scientific Publishing Company

DOI: $10.1142 / \mathrm{S} 0218216516990017$

\title{
Author index \\ Volume 25
}

Abdiel, N., See Frohman

Aicardi, F. \& Juyumaya, 9 (2016) 1641001 J., Tied links

An, B. H.,

Automorphisms of braid groups on orientable surfaces

Bardakov, V. G., Jablan, 9 (2016) 1641002 S. \& Wang, H.,

Monoid and group of pseudo braids

Bataineh, K., Type-two invariants for knots in the solid torus

Biswas, I. \& Gammelgaard, N. L.

Vassiliev invariants from symmetric spaces

Björklund, J., Flexible isotopy classification of flexible links

Bobb, M., Kennedy, S., 6 (2016) 1650034 Wong, H. \& Peifer, D., Roger and Yang's Kauffman bracket arc algebra is finitely generated

Brugallé, E., Koseleff, P.V. \& Pecker, D.,

On the lexicographic degree of two-bridge knots

Brugallé, E., Koseleff, P.V. \& Pecker, D.,

Untangling trigonal diagrams

Calcut, J. S. \& Metcalf- 8 (2016) 1650046 Burton, J. R.,

Double branched covers of theta-curves

8 (2016) 1650051

8 (2016) 1650049

7 (2016) 1650044

7 (2016) 1650043
5 (2016) 1650022

$10(2016) 1650055$

Caprau, C., Movie moves for singular link cobordisms in 4dimensional space

Carrega, A.,

The Tait conjecture in $S^{1} \times S^{2}$

Carter, J. S., Fractal simplices

Casali, M. R., Cristofori, P. \& Gagliardi, C. PL 4-manifolds admitting simple crystallizations: framed links and regular genus

Cha, J. C. \& Tanaka, T., Classical homological invariants are not determined by knot Floer homology and Khovanov homology

Champanerkar, A., Kof- 3 (2016) 1640001 man, I. \& Purcell, J. S., Density spectra for knots

Chmutov, S., Jablan, S., 9 (2016) 1641004 Karvounis, K. \& Lambropoulou, S.,

On the link invariants from the YokonumaHecke algebras

Cho, J.

Connected sum of representations of knot groups - Erratum

Chrisman, M., Virtual covers of links

Clark, W. E., Saito, M. \& 5 (2016) 1650024 Vendramin, L., Quandle coloring and cocycle invariants of composite knots and abelian extensions 5 (2016) 1692001

2 (2016) 1650013

11 (2016) 1650063

9 (2016) 1641003

1 (2016) 1650005

7 (2016) 1650039

8 (2016) 1650052 
Coquereaux, R. \& Zuber, J.-B.,

Maps, immersions and permutations

Cristofori, P., See Casali

de Mattos, D. See Theodoro de Lima

Diamantis,

I., Lambropoulou, S. \& Przytycki, J. H.,

Topological steps toward the Homflypt skein module of the lens spaces $L(p, 1)$ via braids

Diamantis,

I., Lambropoulou, S. \&

Przytycki, J.,

Topological steps toward the Homflypt skein module of the lens spaces $L(p, 1)$ via braids

Dougherty, A. L., Park, H. \& Yetter, D. N., On 2-dimensional Dijkgraaf-Witten theory with defects

Du, K.

Unstabilized dual Heegaard splittings of 3manifolds

Duzhin, F. \& Le, Q.-N., The group of spheretrivial braids

Duzhin, V. S. \& Vasilyev, N. N.,

Asymptotic behavior of normalized dimensions of standard and strict Young diagrams growth and oscillations

Edwin Clark, W. \& Saito, M.,

Algebraic properties of quandle extensions and values of cocycle knot invariants

Egsgaard, J. K. \& Jørgensen, S. F.,

The homological content of the Jones representations at $q=-1$
8 (2016) 1650047

1 (2016) 1650005

1 (2016) 1650001

14 (2016) 1650084

14 (2016) 1650084

5 (2016) 1650021

6 (2016) 1650032

12 (2016) 1642001

12 (2016) 1642002

14 (2016) 1650080

11 (2016) 1650062 See Radović Determinant of links, spanning trees, and a theorem of Shank

Gerdes, P.

3 (2016) 1640003

7 (2016) 1650037

A.,

Nullification numbers of knots with up to 10 crossings

Farinati, M. A. \& García 13 (2016) 1650070 Galofre, J.,

Link and knot invariants from non-abelian Yang-Baxter 2-cocycles

Fedoseev, D. A. \& Manturov, V. O.,

Parities on 2-knots and 2-links

Fish, A. \& Keyman, E., Classifying links under fused isotopy

Frohman, C. \& Abdiel, 4 (2016) 1650016 N.,

Frobenius algebras derived from the Kauffman bracket skein algebra

Funahashi, Y., Nakanishi, Y. \& Satoh, S.,

A note on the OU sequences of a 2-bridge knot

Gagliardi, C. See Casali

Galashin, P. \& Panina, G.

Manifolds associated to simple games

Gallagher, K., See Goryunov

Gammelgaard, N. L., See Biswas

García Galofre, J., See Farinati

14 (2016) 1650079

7 (2016) 1650042

1 (2016) 1650005

12 (2016) 1642003

12 (2016) 1642004

10 (2016) 1650055

13 (2016) 1650070

9 (2016) 1641005

9 (2016) 1641006 
Gobet, T.,

Noncrossing par-

titions, fully commuta-

tive elements and bases of the Temperley-Lieb algebra

Gonzaléz Acuña, F., See Rodríguez-Viorato

Goryunov, V. \& Gallagher, K.,

On planar caustics

Gukov, S., See Elliot

Guo, Q. \& Liu, Q., Connectivity of certain simplicial complexes associated to a handlebody

Ham, J.-Y. \& Lee, J., An explicit formula for the $A$-polynomial of the knot with Conway's notation $C(2 n, 3)$

Ham, J.-Y. \& Lee, J., The volume of hyperbolic cone-manifolds of the knot with Conway's notation $C(2 n, 3)$

Henrich, A., Jablan, S. \& Johnson, I.,

The signed weighted resolution set is not a complete pseudoknot invariant

Igram, S., Millett, K. C. \& Panagioutou, E.,

Resolving critical degrees of entanglement in Olympic ring systems

Imabeppu, T., On Sawollek polynomials of checkerboard colorable virtual links

Inoue, A. \& Shimizu, R., A subspecies of region crossing change, region freeze crossing change

Ishizeki, A. \& Nagasawa, T.,

The invariance of decomposed Möbius energies under inversions with center on curves
6 (2016) 1650035

2 (2016) 1650012

12 (2016) 1642004

3 (2016) 1640003

11 (2016) 1650064

10 (2016) 1650057

6 (2016) 1650030

9 (2016) 1641007

14 (2016) 1650081

2 (2016) 1650010

14 (2016) 1650075

2 (2016) 1650009
Ito, T.,

Framing functions and a strengthened version of Dehn's lemma

Jablan, S., Kauffman, L. 9 (2016) 1641008 H. \& Lopes, P.,

Delta diagrams

Jablan, S., See Bardakov

Jablan, S., See Chmutov

Jablan, S., See Henrich

Jablan, S., See Kappraff

Jablan, S., See Radović

Jablan, S., See Zeković

Jabłonowski, M., On a banded link presentation of knotted surfaces

Jeong, M.-J. \& Kim, D.G.,

Clasp-pass moves and Kauffman polynomials of virtual knots

Jeong, M.-J., A zero polynomial of virtual knots

Jiménez Pascual, A., On lassos and the Jones polynomial of satellite knots

Johnson, I., See Henrich

Jørgensen, S. F., See Egsgaard

Juyumaya, J., See Aicardi

Kalinin, N. \& Shkolnikov, M.,

Sandpiles on the heptagonal tiling

Kamada, N. \& Kamada, 9 (2016) 1641011 S.,

Double coverings of twisted links

Kamada, S., See Kamada

Kanenobu, T., Band surgery on knots and links, III
6 (2016) 1650031

9 (2016) 1641002

9 (2016) 1641004

9 (2016) 1641007

9 (2016) 1641009

9 (2016) 1641006

9 (2016) 1641010

1 (2016) 1550078

2 (2016) 1650011

9 (2016) 1641007

11 (2016) 1650062

9 (2016) 1641001

12 (2016) 1642005
3 (2016) 1640004

8 (2016) 1650045
9 (2016) 1641011

10 (2016) 1650056 
Kappraff, J., Radović, L. 9 (2016) 1641009 \& Jablan, S.

Meanders, knots, laby-

rinths and mazes

Karvounis, K., See Chmutov

Karvounis, K., Enabling computations for link invariants coming from the Yokonuma-Hecke algebras

Katsir, R. Elliptic associators and the LMO functor

Kauffman, L. H. \& Sil- 3 (2016) 1640005 vero, M.,

Alexander-Conway polynomial state model and link homology

Kauffman, L. H., See Jablan

Kauffman, L., See Zeković

Kennedy, S., See Bobb

Keyman, E., See Fish

Khovanov, M., Hopfological algebra and categorification at a root of unity: The first steps

Kim, D.-G. See Jeong

Kim, S. \& Manturov, V. O.,

On groups $G_{n}^{k}$, braids and Brunnian braids

Kleptsyn, V. \& Smirnov, E.

Ribbon graphs and bialgebra of Lagrangian subspaces

Kofman, I., See Champanerkar

Kohli, B.-M.,

On the Links-Gould invariant and the square of the Alexander polynomial

Kohno, T.,

Higher holonomy of formal homology connections and braid cobordisms

9 (2016) 1641010

6 (2016) 1650034

7 (2016) 1650042

3 (2016) 1640006

8 (2016) 1650045

2 (2016) 1650006
Kong, T. See Mellor

4 (2016) 1650019

Koseleff, P.-V. \& Pecker, 13 (2016) 1650074 D., Harmonic knots

Koseleff, P.-V.,

Koseleff, P.-V., See Brugallé

Kotorii, Y., A relation between Milnor's $\quad \mu$-invariants and HOMFLYPT polynomials

Kreinbihl, J., Martin, R., Murphy, C., Renn, M. \& Townsend, J.,

Alternation by region crossing changes and implications for warping span

13 (2016) 1650078

Lambropoulou, S.,

Lambropoulou, S.,

Lambropoulou, S., See Diamantis

Lamey, K. R., Silver, D. S. \& Williams, S. G.,

Vertex-colored graphs, bicycle spaces and Mahler measure

Le, Q.-N.,

Lee, D. \& Sakuma, M., Homotopically equivalent simple loops on 2-bridge spheres in Heckoid orbifolds for 2bridge links (I)

12 (2016) 1642006

Lee, D. \& Sakuma, M.,

equivalent simple loops on 2-bridge spheres in Heckoid orbifolds for 2bridge links (II)

12 (2016) 1642007

Lee, D. \& Sakuma, M., Parabolic generating pairs of genus-one

Lee, H. J. \& Takioka, H., cable links and Whitehead doubles

Lee, J., See Brugallé See Chmutov See Diamantis See Duzhin Homotopically 2-bridge knot groups On the arc index of

10 (2016) 1650057

7 (2016) 1650043

7 (2016) 1650044

13 (2016) 1650072

13 (2016) 1650073

9 (2016) 1641004

14 (2016) 1650084

14 (2016) 1650084

6 (2016) 1650033

12 (2016) 1642001

11 (2016) 1650067

11 (2016) 1650066

5 (2016) 1650023

7 (2016) 1650041

See Ham 
Lee, J., See Ham

Lewald, A., See Mellor

Li, C.

Temperley-Lieb algebra approach to Catalan states of generalized crossing and lattice crossing

Lightfoot, A., On invariants of link maps in dimension four

Liu, Q., See Guo

Lopes, P., See Jablan

Lozano, M. T. \& Montesinos-Amilibia, J. M.,

On continuous families of geometric Seifert conemanifold structures

Lozano-Rojo, Á. \& Vigara, R.,

Representing knots by filling Dehn spheres

Manturov, V. O., See Fedoseev

Manturov, V. O., See Kim

Manturov, V. O., New parities and coverings over free knots

Marengon, M., On $d$-invariants and generalized Kanenobu knots

Markarian, N., Weyl $n$-algebras and the Kontsevich integral of the unknot

Martin, R., See Kreinbihl

Masai, H., On the number of commensurable fibrations on a hyperbolic 3manifold

Mellor, B., Kong, T., 4 (2016) 1650019 Lewald, A. \& Pigrish, V.,

Colorings, determinants and Alexander polynomials for spatial graphs
6 (2016) 1650030

4 (2016) 1650019

5 (2016) 1650027

11 (2016) 1650060

11 (2016) 1650064

9 (2016) 1641008

14 (2016) 1650083

4 (2016) 1650018

14 (2016) 1650079

13 (2016) 1650078

11 (2016) 1650077

8 (2016) 1650048

12 (2016) 1642008

13 (2016) 1650073

5 (2016) 1650028
Mellor, B.,

Alexander and writhe polynomials for virtual knots

Meshveliani, S. D., On a K5 problem by S. V. Duzhin

Metcalf-Burton, J. R., See Calcut

Mikhalkin, G. \& Orevkov, 12 (2016) 1642010 S.,

Real algebraic knots and links of small degree

Millett, K. C., See Igram

Millett, K. C.,

Knots in knots: A study of classical knot diagrams

Mizusawa, A. \& Murakami, J.,

Yokota type invariants derived from nonintegral highest weight representations of $\mathcal{U}_{q}\left(s l_{2}\right)$

Mohamad, A. \& Yashiro, 10 (2016) 1650053 T.,

On construction of surface-knots

Montemayor, A., See Ernst

Montesinos-Amilibia, J. 14 (2016) 1650083 M.,

See Lozano

Montesinos-Amilibia, J. 3 (2016) 1640007 M.,

On some groups related with Fox-Artin wild $\operatorname{arcs}$

Mou, G. \& Pasechnik, D. 12 (2016) 1642011 V.,

Edge-dominating cycles, $k$-walks and Hamilton prisms in $2 K_{2}$-free graphs

Moutuou, E. M., See Elhamdadi

Murakami, J., See Mizusawa

Murao, T., On bind maps for braids

Murphy, C., See Kreinbihl
8 (2016) 1650050

12 (2016) 1642009

8 (2016) 1650046

14 (2016) 1650081

9 (2016) 1641013

10 (2016) 1650054

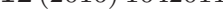

列


Nagasawa, T., See Ishizeki

Nakamura, I., On addition of 1handles with chart loops to 2-dimensional braids

Nakamura, T., Nakanishi, Y. \& Satoh, S. 11-Colored knot diagram with five colors

Nakanishi, Y., See Funahashi

Nakanishi, Y., See Nakamura

Nasybullov, T., Classification of fused links

Nelson, S. \& Rivera, P., Bikei invariants and gauss diagrams for virtual knotted surfaces

Oberti, C. \& Ricca, R. L., 6 (2016) 1650036 On torus knots and unknots

Orevkov, S., See Mikhalkin

Owczarek, R., Quantum mechanics for quantum computing

Ozsváth, P., Stipsicz, A. I. 1 (2016) 1650003 \& Szabó, Z.,

Knot lattice homology in $L$-spaces

Panagioutou, E., See Igram

Pancholi, D. M. \& Pandit, 13 (2016) 1650069 S., Geometry of Legendrian knots

Pandit, S. See Pancholi

Panina, G., See Galashin

Park, H. See Dougherty

Pasechnik, D. V., See Mou

Pecker, D. See Brugallé

Pecker, D., See Brugallé

Pecker, D., See Koseleff

4 (2016) 1650017

13 (2016) 1671001

4 (2016) 1650017

14 (2016) 1650076

3 (2016) 1640008

12 (2016) 1642010

3 (2016) 1640009

12 (2016) 1642011

7 (2016) 1650043

7 (2016) 1650044

13 (2016) 1650074

\begin{tabular}{l|l}
$\mathbf{2}(2016) 1650009$ & Peifer, D., \\
& See Bobb \\
& Perko, K. A., \\
& Historical high- \\
& lights of non-cyclic knot \\
& theory
\end{tabular}

Petronio, C. \& Zanellati, A.,

Algorithmic simplification of knot diagrams: New moves and experiments

Pigrish, V., See Mellor

Przytycki, J. H. \& Yang, S. Y.,

Annihilation of torsion in homology of finite $m$ AQ quandles

Przytycki, J. H., See Diamantis

Przytycki, J. H., On Slavik Jablan's work on 4-moves

Przytycki, J., See Diamantis

Purcell, J. S., See Champanerkar

6 (2016) 1650034

3 (2016) 1640010

10 (2016) 1650059

4 (2016) 1650019

12 (2016) 1642012

14 (2016) 1650084

9 (2016) 1641014

14 (2016) 1650084

3 (2016) 1640001

3 (2016) 1640012 makovitch, A. N. Knot invariants arising from homological operations on Khovanov homology

Putyra, K. K.,

3 (2016) 1640011

On a triply graded Khovanov homology

Radović,

L., 9 (2016) 1641006

Gerdes, P., Jablan, S. \&

Sazdanovic, R.,

Plaited polyhedra: A knot theory point of view

Radović, L., See Kappraff

Renn, M., See Kreinbihl
Ricca, R. L., See Oberti

Rimanyi, R. \& Varchenko, A.,

Dynamical GelfandZetlin algebra and equivariant cohomology of grassmannians
9 (2016) 1641009

13 (2016) 1650073

6 (2016) 1650036

12 (2016) 1642013 
Rivera, P., See Nelson

Robert, L.-H., Categorification of the colored $\mathfrak{s l}_{3}$-invariant

Rodríguez-Viorato, J. \& Gonzaléz Acuña, F., On pretzel knots and Conjecture $\mathbb{Z}$

Saito, M., See Clark

Saito, M., See Edwin Clark

Saito, T. Examples of nested essential free tangles

Sakata, N., Veering structures of the canonical decompositions of hyperbolic fibered two-bridge link complements

Sakuma, M., See Lee

Sakuma, M., See Lee

Sakuma, M., See Lee

Sakurai, M., An affine index polynomial invariant and the forbidden move of virtual knots

Salvetti, M. \& Serventi, M.,

Arrangements of lines and monodromy of associated Milnor fibers

Satoh, S., See Funahashi

Satoh, S., See Nakamura

Sazdanovic, R., See Radović

Sazdanovic, R., See Zeković

Scanlon, L. A., Study of knots in material culture

Schneider, G. R., Foliated compressing discs for Legendrian rational tangles
3 (2016) 1640008

7 (2016) 1650038

2 (2016) 1650012

5 (2016) 1650024

14 (2016) 1650080

4 (2016) 1650020

4 (2016) 1650015

11 (2016) 1650066

11 (2016) 1650067

5 (2016) 1650023

7 (2016) 1650040

12 (2016) 1642014

13 (2016) 1671001

4 (2016) 1650017

9 (2016) 1641006

9 (2016) 1641010

9 (2016) 1641015

6 (2016) 1650029
Serventi, M., See Salvetti

Shimizu, R., See Inoue

Shkolnikov, M., See Kalinin

Shumakovitch, A. N., $\quad 3$ (2016) 1640012 See Putyra

Silver, D. S., See Lamey

Silvero, M., See Kauffman

Silvero, M., Strongly quasipositive links with braid index 3 have positive Conway polynomial

Singh, M., Classification of flat connected quandles

Smirnov, E., See Kleptsyn

Soret, M. \& Ville, M., Lissajous and Fourier knots

Stipsicz, A. I., See Ozsváth

Stošić, M., See Zeković

Stošić, M., Slavik Jablan's last notes on the ChernSimons invariants of knots

Szabó, Z., See Ozsváth

Tagami, K.,

Bar-Natan's geometric complex and a categorification of the DyeKauffman-Miyazawa polynomial

Takaoka, K., $\mathrm{PN}$ sequences obtained from signs of crossings of knot diagrams

Takemura, A., On a relation between conway polynomials of $(m, n)$ - and $(n, m)$ Turk's head links

Takioka, H., See Lee
12 (2016) 1642014

14 (2016) 1650075

12 (2016) 1642005

6 (2016) 1650033

3 (2016) 1640005

12 (2016) 1642015

13 (2016) 1650071

12 (2016) 1642006

5 (2016) 1650026

1 (2016) 1650003

9 (2016) 1641010

9 (2016) 1641016

1 (2016) 1650003

1 (2016) 1550076

10 (2016) 1650058

14 (2016) 1650082

7 (2016) 1650041 
Tanaka, K.,

A note on the local relations in geometric formalism of Khovanov homology theory

Tanaka, T., See Cha

Theodoro de Lima, J. R. \& de Mattos, D., Ordering homotopy string links over surfaces

Townsend, J., See Kreinbihl

Tran, A. T., Adjoint twisted Alexander polynomials of genus one two-bridge knots

Tran, A. T. Character varieties of $(-2,2 m+1,2 n)$-pretzel links and twisted Whitehead links

Tran, A. T. Nonabelian representations and signatures of double twist knots

Tsau, C. M., On the topology of the coefficients of the Alexander-Conway polynomials of knots

Varchenko, A., See Rimanyi

Vasilyev, N. N., See Duzhin

Vassiliev, V. A., Linking numbers in non-orientable 3-manifolds

Vendramin, L., See Clark
1 (2016) 1620001

7 (2016) 1650039

1 (2016) 1650001

13 (2016) 1650073

11 (2016) 1650065

2 (2016) 1650007

3 (2016) 1640013

2 (2016) 1650008

12 (2016) 1642013

12 (2016) 1642002

12 (2016) 1642016

5 (2016) 1650024
Vigara, R.,

4 (2016) 1650018

See Lozano-Rojo

Ville, M.,

5 (2016) 1650026

See Soret

Wada, K.,

Note on Milnor's $\bar{\mu}$ - and $\mu^{*}$-invariants of links

Wang, H., See Bardakov

Wigderson, Y., The Bar-Natan theory splits

Williams, S. G., See Lamey

Wong, H., See Bobb

Yang, S. Y., See Przytycki

Yashiro, T., See Mohamad

Yashiro, T., Pseudo-cylces of surface-knots

Yetter, D. N., See Dougherty

6 (2016) 1620002

9 (2016) 1641002

4 (2016) 1650014

6 (2016) 1650033

6 (2016) 1650034

12 (2016) 1642012

10 (2016) 1650053

13 (2016) 1650068

Yokota, Y., On the cusp shape of hyperbolic knots

Zanellati, A., See Petronio

Zeković A., Jablan, S., Kauffman, L., Sazdanovic, R. \& Stošić, M. Unknotting and maximum unknotting numbers

Zhang, L.,

9 (2016) 1641005 See Ge

Zuber, J.-B., 8 (2016) 1650047
5 (2016) 1650021

5 (2016) 1650025

10 (2016) 1650059

9 (2016) 1641010 